Jakob Usemann, Lars Garten, Christoph Bührer, Christof Dame* and Malte Cremer

\title{
Fresh frozen plasma transfusion - a risk factor for pulmonary hemorrhage in extremely low birth weight infants?
}

DOI 10.1515/jpm-2016-0309

Received September 19, 2016. Accepted December 9, 2016. Previously published online February 14, 2017.

\section{Abstract}

Aim: To evaluate risk factors for pulmonary hemorrhage $(\mathrm{PH})$ in extremely low birth weight infants (ELBW) taking into consideration coagulation screens, platelet counts, transfusion of fresh frozen plasma (FFP), and platelet concentrates prior to $\mathrm{PH}$.

Patients and methods: A retrospective case-control study consisting of 20 ELBW infants with $\mathrm{PH}$ and 40 matched controls. Coagulation screens, platelet counts at birth and at onset of $\mathrm{PH}$, and transfusion frequencies prior to $\mathrm{PH}$ were compared to case-controls at birth and 24-96 h after birth.

Results: While the initial platelet counts, fibrinogen concentrations, and international normalized ratios were similar in PH infants and controls, the activated partial prothrombin time was prolonged $(\mathrm{P}=0.05)$. Compared to $28 \%$ of case controls ( $\mathrm{P}<0.05), 55 \%$ of infants with later $\mathrm{PH}$ received $\mathrm{FFP}$ prior to $\mathrm{PH}$. Platelet counts were significantly lower at onset of $\mathrm{PH}$ (median $81 / \mathrm{nL}$; range: $37-236 / \mathrm{nL})$ compared to controls $(166 / \mathrm{nL} ; 27-460 / \mathrm{nL}$; $\mathrm{P}<0.005)$. Multivariate analysis indicated a lack of antenatal steroids, supplemental oxygen, and transfusion of FFP as independent risk factors for $\mathrm{PH}$.

Conclusion: Prolonged activated partial thromboplastin time (aPTT) might be associated with $\mathrm{PH}$. PH does not primarily depend upon severe thrombocytopenia. A developmental mismatch in hemostasis by transfusion of adult donor plasma should be considered a risk factor for $\mathrm{PH}$.

\footnotetext{
*Corresponding author: Christof Dame, MD, Department of Neonatology, Charité - Universitätsmedizin Berlin, Augustenburger Platz 1, D-13353 Berlin, Germany, Tel.: + 4930 450559006, Fax: + 4930 450559906, E-mail: christof.dame@charite.de Jakob Usemann: Department of Neonatology, Charité Universitätsmedizin Berlin, Germany; and Department of Pediatrics, University of Basel Children's Hospital, Basel, Switzerland Lars Garten, Christoph Bührer and Malte Cremer: Department of Neonatology, Charité - Universitätsmedizin Berlin, Germany
}

Keywords: Bleeding; coagulation disorder; extremely low birth weight; fresh frozen plasma; platelet transfusion; thrombocytopenia.

\section{Introduction}

In extremely low birth weight infants (ELBW; <1000 g), pulmonary hemorrhage $(\mathrm{PH})$ is a rare but life-threatening event associated with mortality rates as high as 50\%-68\% [1-3]. The risk of $\mathrm{PH}$ is inversely related to gestational age. Numerous findings are associated with $\mathrm{PH}$, such as intrauterine growth restriction, lung hypoplasia, sepsis, coagulopathy, thrombocytopenia, surfactant application, intraventricular hemorrhage (IVH), and patent ductus arteriosus (PDA) [1, 2]. While previous studies indicate that antenatal glucocorticoids may be protective against $\mathrm{PH}$ [4], putative adverse effects of transfusions of adult red blood cells, platelets, or plasma are a matter of discussion. In particular, the routine performance of coagulation screens at admission causes an increased use of fresh frozen plasma (FFP), although their clinical benefit has to be confirmed in randomized clinical trials [3]. Thus, we reassessed risk factors for $\mathrm{PH}$ with specific emphasis on routine coagulation screens, including platelet counts and transfusion of blood products.

\section{Patients and methods}

This retrospective case-control study included 20 consecutive ELBW infants with PH admitted to our neonatal intensive care unit (NICU) between 2002 and 2012. PH was defined by the presence of frank tracheal blood requiring prompt intervention (e.g. red blood cell transfusions, increased respiratory support) and by multi-lobular opacity on chest X-rays [2,5]. The cohort was compared with gestational age and weight-matched controls $(n=40)$.

We considered two different time points of blood sampling in infants with PH and case-controls. Immediately after birth, ELBW infants had routine blood tests, including full blood cell count, interleukin-6 (IL-6), and coagulation screens. The specimens were taken via direct venipuncture or through a non-heparinized umbilical line. At the second time point, a full blood cell count was performed at least once 24-96 h after birth, according to standard procedure. In 
patients with $\mathrm{PH}$, another examination of full blood count was initiated as soon as PH was recognized.

Blood cell counts were determined using the automated analyzer XE-2100 ${ }^{\mathrm{TM}}$ (Sysmex Corporation, Kobe, Japan). Plasma IL-6 concentrations were measured by means of an automated immunoassay (COBAS 6000 or COBAS 8000, Roche Diagnostics, Mannheim, Germany). Coagulation screens, including activated partial thromboplastin time (aPTT), international normalized ratio (INR), and fibrinogen concentration, were performed on the COBAS t 411 coagulation analyzer (Roche Diagnostics, Mannheim, Germany). Thrombocytopenia was defined by a platelet count $<150 /$ nL. Fibrinogen, INR, and aPTT were interpreted considering age-dependent reference values [6, 7]. Abnormal values were defined as those outside of two standard deviations of the mean (aPTT $>64 \mathrm{~s}$, INR $>2.7$, fibrinogen $<0.71 \mathrm{~g} / \mathrm{L}$ ). Early-onset infection was diagnosed by increased IL-6 concentration ( $>100 \mathrm{ng} / \mathrm{L}$ ) [8]. Septicemia was defined by positive blood culture within $48 \mathrm{~h}$. Echocardiographic examination for PDA included assessment of ductal shunt direction by color Doppler and measurement of the minimal internal ductal diameter in B-mode on the $4^{\text {th }}$ or the $5^{\text {th }}$ day of life. The left-atrium-to-aortic-root ratio was determined using the M-mode in the parasternal long axis view. Ibuprofen treatment was only initiated in hemodynamically significant PDA with left-to-right shunt, according to the following conditions: (1) respiratory setback with supplementary oxygen $>30 \%$ and/or invasive ventilation, (2) left atrium to aortic root ratio $\geq 1.4$ or a PDA diameter $\geq 2.5 \mathrm{~mm}$. Ibuprofen (Pedea) was given with three doses of 10,5 , and $5 \mathrm{mg} / \mathrm{kg}$ per dose at 24-h intervals. Cerebral ultrasound examinations were performed on the $1^{\text {st }}, 3^{\text {rd }}$, and $7^{\text {th }}$ day after birth. In patients with $\mathrm{PH}$, cerebral ultrasound examination was additionally performed after diagnosis of PH. The grade of intraventricular hemorrhage (IVH) was defined according to Papile et al. [9]. Indomethacin treatment for IVH prevention was initiated immediately after birth if ELBW infants were at high risk for IVH. According to our internal standard operating procedure, we calculated a risk score based on previously published data $[10,11]$, including completion of prenatal steroids for lung maturation [12], gestational age, sex [13], multiple birth [14], and IL-6 concentration at birth [15]. Indomethacin was used at a dose of $0.1 \mathrm{mg} / \mathrm{kg}$ intravenously for 3 consecutive days if the patients were classified as high risk for IVH. Other bleeding events were defined as bloody staining of oral, nasal, or gastric secretion, puncture site oozing, or macrohematuria.

Platelet transfusions were administered according to previously published recommendations [16]. Briefly, platelets were transfused under the following conditions: (1) ELBW infants with acute major bleeding and a platelet count below 100/nL. (2) Non-bleeding ELBW infants, if the platelet count fell below 50/nL in the first week of life. (3) ELBW infants with concurrent coagulopathy or signs of minor bleeding and a platelet count of 50-99/nL in the first week. Only single donor (apheresis-derived), leukoreduced, and irradiated platelet concentrates were transfused. The transfusion volume was $10 \mathrm{~mL} / \mathrm{kg}$ body weight, administered over $1-2 \mathrm{~h}$, preferentially via a peripheral venous line.

FFP was transfused in infants with abnormal coagulation screens. Plasma packages were produced from whole blood, pooled from various donors, and stored at $-30^{\circ} \mathrm{C}$ until use. Normally, $10-$ $20 \mathrm{~mL} / \mathrm{kg}$ FFP was transfused over approximately $4 \mathrm{~h}$.

For this study, we recorded the number and time points of FFP and platelet concentrate transfusions in the first week after birth. In patients with $\mathrm{PH}$, the transfusion of blood products was only recorded, when given prior to the onset of PH (minimum clearance
$24 \mathrm{~h}$ ). We analyzed the frequency (none/one/more than one) of FFP/ platelet concentrates transfused.

For statistical analysis of platelet counts and coagulation screens, the initial blood examination at birth (first time point) was considered. Platelet counts were analyzed at onset of PH and compared to the second routine blood examination performed within 24$96 \mathrm{~h}$ postnatally in case-controls (second time point). The difference of the individual platelet counts between the first and second time point were compared using the Wilcoxon matched-pairs signed-rank test. To assess the relation between risk factors and $\mathrm{PH}$, multivariate logistic regression was used [yielding adjusted odds ratio (OR) with 95\% confidence interval (CI)]. Variables significantly associated with $\mathrm{PH}$ in the univariate model (FFP transfusion, fraction of inspired oxygen, antenatal steroids) were included in the multivariate model. As published data also suggests an association between sex and $\mathrm{PH}$ in ELBW infants, this variable was also included in the multivariate model. Statistics were performed with Stata ${ }^{\circledR}$ (Stata Statistical Software: release 13. STATA Cooperation, College Station, TX, USA), using the $\chi^{2}$-test or the Mann-Whitney $U$-test as appropriate.

\section{Results}

PH was diagnosed in 20 out of 761 (2.6\%) ELBW infants admitted within the 11-year observation period. $\mathrm{PH}$ occurred, in median, on day (d) 3 (range: d2-d5). $\mathrm{PH}$ occurred most frequently within the first $72 \mathrm{~h}$ after birth $(70 \%)$. The demographic and clinical data are summarized in Table 1. The overall short-term outcome in the $\mathrm{PH}$ group was poor, including a significantly higher incidence $(55 \%)$ of severe IVH and a mortality rate of $35 \%$ within the first 7 days of life. In infants with $\mathrm{PH}$, steroids were given significantly less often as compared to case-controls $(\mathrm{P}=0.013)$. The frequency of intubation and surfactant application in the delivery room did not differ between infants with $\mathrm{PH}$ and case-controls. At admission to the NICU, the clinical risk index for babies (CRIB) score and the incidence of early-onset infection were similar in both groups. The median concentration of IL-6 did not differ between infants with PH $(149 \mathrm{pg} / \mathrm{mL}$, range 1-90,000) and controls $(223 \mathrm{pg} / \mathrm{mL}$, range 5-66,600; $\mathrm{P}=0.269)$. Infants with later $\mathrm{PH}$ had a significantly higher concentration of supplemental oxygen at admission to NICU. The type of mechanical ventilation (conventional or high frequency oscillation) and rate of respiratory support (continuous positive airway pressure, CPAP) treatment at onset of $\mathrm{PH}$ were similar compared to case-controls. The frequency of indomethacin treatment for the prevention of IVH did not differ significantly between the groups. $\mathrm{PH}$ was associated with a significantly higher incidence of IVH, both considering any grade of IVH $(\mathrm{P}=0.003)$ or only severe IVH (stage $\geq \mathrm{III}^{\circ}, \mathrm{P}=0.002$ ). In 10 out of 11 patients with $\mathrm{PH}$, severe IVH was first diagnosed after the occurrence of $\mathrm{PH}$. 
Table 1: Demographic and clinical characteristics of both groups of patients.

\begin{tabular}{|c|c|c|c|}
\hline & $\begin{array}{r}\text { Pulmonary } \\
\text { hemorrhage }(n=20)\end{array}$ & $\begin{array}{r}\text { Case-controls } \\
(n=40)\end{array}$ & P-value \\
\hline Females & $5(25 \%)$ & $15(37.5 \%)$ & 0.33 \\
\hline Singletons & $10(50 \%)$ & $27(67.5 \%)$ & 0.26 \\
\hline Birth weight (g) & $713(488-990)$ & $737(438-1024)$ & 0.86 \\
\hline Small for gestational age & $8(40 \%)$ & $16(40 \%)$ & 0.78 \\
\hline Gestational age (weeks + days) & $26+2(24+1-30+0)$ & $26+0(24+0-31+5)$ & 0.52 \\
\hline Antenatal steroids & $14(70 \%)$ & 38 (95\%) & 0.013 \\
\hline Intubation in delivery room & $17(85 \%)$ & $38(95 \%)$ & 0.17 \\
\hline Surfactant replacement therapy & $19(95 \%)$ & 37 (92.5\%) & 1.0 \\
\hline Fraction of inspired oxygen (\%) & $58(21-73)$ & $40(21-74)$ & 0.003 \\
\hline CRIB score & $7(1-17)$ & $6.5(2-11)$ & 0.63 \\
\hline Early-onset infection & $11(55 \%)$ & $27(67.5 \%)$ & 0.90 \\
\hline Positive blood culture at birth & $4(20 \%)$ & $2(5 \%)$ & 0.09 \\
\hline Platelet count $(/ \mathrm{nL})$ at birth & $170(61-446)$ & $183(45-443)$ & 0.51 \\
\hline Platelet count $(/ \mathrm{nL})$ at onset of $\mathrm{PH}$ or within first $96 \mathrm{~h}$ (controls) & $81(37-236)$ & $166(27-460)$ & 0.002 \\
\hline Conventional mechanical ventilation & $15(75 \%)$ & $25(62.5 \%)$ & 0.67 \\
\hline High-frequency oscillatory ventilation & $1(5 \%)$ & $2(5 \%)$ & \\
\hline CPAP respiratory support & $4(20 \%)$ & $12(30 \%)$ & \\
\hline Early indomethacin ( $<12 \mathrm{~h}$ of life) for IVH prevention & $1(5 \%)$ & $8(20 \%)$ & 0.12 \\
\hline PDA & $18(90 \%)$ & $33(83 \%)$ & 0.58 \\
\hline Ibuprofen treatment for hemodynamically significant PDA & $2(10 \%)$ & $10(25 \%)$ & 0.17 \\
\hline IVH (all grades) & $12(60 \%)$ & $8(20 \%)$ & 0.003 \\
\hline Severe IVH (grade $\geq$ III) & $11(55 \%)$ & $6(15 \%)$ & 0.002 \\
\hline Minor bleeding ${ }^{b}$ & $3(15 \%)$ & 0 & 0.033 \\
\hline Death $(\leq \mathrm{d} 7)$ & $7(35 \%)$ & $4(10 \%)$ & 0.03 \\
\hline Death $(>d 7)$ & $1(5 \%)$ & $5(12.5 \%)$ & 0.36 \\
\hline Overall death during hospital stage & $8(40 \%)$ & $9(22.5 \%)$ & 0.16 \\
\hline
\end{tabular}

Data are presented as median (range) or number (\%). a Maximum fraction of inspired oxygen during the first $24 \mathrm{~h}$ after birth to achieve an oxygen saturation level $>85 \%$. ${ }^{\mathrm{b}} \mathrm{Minor}$ bleeding was defined as one or more of the following signs: blood staining of oral, nasal, or gastric secretion, puncture site oozing, or macrohematuria. CRIB, Clinical risk index for babies; CPAP, continuous positive airway pressure; IVH, intraventricular hemorrhage; PDA, patent ductus arteriosus; d, day of life.

In case-controls, IVH was diagnosed, in median, on day 3 (range d1-d3) in six infants. Other bleedings occurred in three infants with PH (two infants with gastrointestinal bleeding, one infant with macrohematuria), but not in controls. Among them, gastrointestinal bleeding was diagnosed contemporaneously to $\mathrm{PH}$; macrohematuria occurred in one patient 7 days after the appearance of $\mathrm{PH}$. Overall, the incidence of other bleeding events was significantly higher in $\mathrm{PH}$ patients $(\mathrm{P}=0.033)$. Early mortality $(\leq \mathrm{d} 7)$ was higher after $\mathrm{PH}$ compared to case-controls $(\mathrm{P}=0.03)$, while the overall in-hospital mortality rate was not different.

The incidence of thrombocytopenia and median platelet count at birth did not differ between both groups (platelet count $170 / \mathrm{nL}$ vs. $183 / \mathrm{nL}$, Figure 1). In infants with $\mathrm{PH}$, platelet counts decreased at onset of $\mathrm{PH}$ and were significantly lower (platelet count $81 / \mathrm{nL}$ vs. 166/ $\mathrm{nL}, \mathrm{P}=0.002$ ) than in case-controls at a comparable time

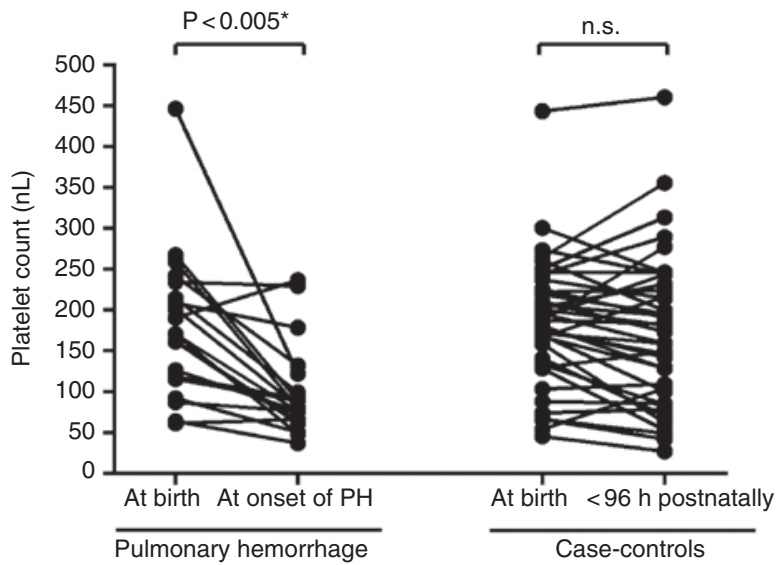

Figure 1: Individual platelets counts of each patient suffering from pulmonary hemorrhage $(\mathrm{PH})(\mathrm{n}=20)$ and matched case-controls $(n=40)$ at birth and at onset of PH or within 96 h postnatally, respectively. *Results obtained with the Wilcoxon matched-pairs signed-rank test. 
point (24-96 h postnatally). In contrast, platelet counts in case-controls did not significantly change during the observation period $(\mathrm{P}=0.405)$. Only $10 \%$ of the infants displayed platelet counts $<50 / \mathrm{nL}$ at the onset of $\mathrm{PH}$, while in the case-control group, $7.5 \%$ presented platelet counts $<50 /$ nL (Figure 1).

Due to the small sample volume, aPTT measurement could not be performed in one infant with PH. aPTT measurement was missing in nine, and fibrinogen and INR in 11 case-controls, respectively. In infants with $\mathrm{PH}$, the median aPTT was significantly longer (75 s) compared to casecontrols (62 s, P = 0.033). Thirteen infants with PH $(68 \%)$ had prolonged aPTT compared to 12 case-controls (41\%, $\mathrm{P}=0.061)$. The median concentration of fibrinogen did not differ between both groups (PH: median $131 \mathrm{mg} / \mathrm{dL}$, range: 30-378; controls: $178 \mathrm{mg} / \mathrm{dL}, 55-469 ; \mathrm{P}=0.107$ ). Five of twenty infants $(25 \%)$ with $\mathrm{PH}$ had an abnormal fibrinogen concentration compared to two case-controls $(6 \%$, $\mathrm{P}=0.073)$. The INR did not differ significantly between the groups (PH: median INR 2.05, range: 0.92-4.3; controls: 1.6, 0.48-5.4; $\mathrm{P}=0.559$ ).

There was no difference in the administration rate of platelet concentrates in infants with later $\mathrm{PH}$ vs. case-controls: Five out of 20 infants (25\%) were transfused prior to the onset of $\mathrm{PH}$ compared to four out of 40 case-controls $(10 \%, P=0.13)$. In four patients with $\mathrm{PH}$, platelet concentrates were transfused when $\mathrm{PH}$ occurred. In contrast, infants with later hemorrhage received FFP prior to the onset of PH significantly more often (minimum clearance $24 \mathrm{~h}$ ) compared to controls. In patients with later hemorrhage, FFP was transfused in median 1.5 days (range 1-3 days) before PH occurred. Eleven out 20 infants (55\%) received FFP prior to the onset of $\mathrm{PH}$; among them, two patients received FFP twice. Eleven out of 40 case-controls $(27.5 \%)$ were transfused with FFP once in the first 5 days $(\mathrm{P}=0.033)$ (Table 2). We performed uni- and multivariate logistic regressions to study the associations between different risk factors and PH. FFP transfusion remained an independent risk factor for PH [OR 12.9 (95\% CI 2.07-76.94); $\mathrm{P}=0.006$ ], even if adjusted to the application of antenatal steroids, the fraction of inspired oxygen, and sex (Table 3).

\section{Discussion}

A priori, this retrospective single-center case-control study, emphasizing the comparison of coagulation factors and platelet counts, was unlikely to identify a single major risk factor for $\mathrm{PH}$, but we aimed to add our data to the ongoing discussion on preventive strategies [1, 2, 17]. Based on our data and other studies, the following avenues for further clinical recommendations and research might become more obvious.

Table 2: Blood product transfusions in infants with pulmonary hemorrhage and case-controls.

\begin{tabular}{|c|c|c|c|}
\hline & $\begin{array}{r}\text { Pulmonary } \\
\text { hemorrhage }(n=20)\end{array}$ & $\begin{array}{r}\text { Case-controls } \\
(n=40)\end{array}$ & P-value \\
\hline Platelet transfusion prior to onset of $\mathrm{PH}$, or within the first $96 \mathrm{~h}$ (controls) & $5(25 \%)$ & $4(10 \%)$ & 0.13 \\
\hline No FFP transfusion prior to onset of $\mathrm{PH}$, or within the first $96 \mathrm{~h}$ (controls) & $9(45 \%)$ & $29(72.5 \%)$ & \\
\hline $1 \mathrm{FFP}$ transfusion prior to onset of $\mathrm{PH}$, or within the first $96 \mathrm{~h}$ (controls) & $9(45 \%)$ & $14(27.5 \%)$ & \\
\hline$\geq 2$ FFP transfusions prior to onset of $\mathrm{PH}$, or within the first $96 \mathrm{~h}$ (controls) & $2(10 \%)$ & 0 & $0.033^{\mathrm{a}}$ \\
\hline
\end{tabular}

Data are presented as median (range) or number (\%). ${ }^{a}$ Comparison of all three categories. PH, Pulmonary hemorrhage; FFP, fresh frozen plasma.

Table 3: Association between different risk factors and pulmonary hemorrhage.

\begin{tabular}{|c|c|c|c|c|}
\hline & \multicolumn{2}{|c|}{ Univariate association } & \multicolumn{2}{|c|}{ Multivariate association $^{\mathrm{a}}$} \\
\hline & OR $(95 \% \mathrm{Cl})$ & P-value & OR $(95 \% \mathrm{Cl})$ & P-value \\
\hline Female gender & $0.55(0.16-1.84)$ & 0.336 & $0.44(0.09-2.21)$ & 0.324 \\
\hline Antenatal steroids & $0.12(0.02-0.68)$ & 0.016 & $0.11(0.01-0.86)$ & 0.035 \\
\hline Fraction of inspired oxygen (\%) & $1.07(1.02-1.12)$ & 0.003 & $1.06(1.01-1.12)$ & 0.031 \\
\hline $\begin{array}{l}\text { FFP transfusion prior to onset of } \mathrm{PH} \text {, or } \\
\text { within the first } 96 \mathrm{~h} \text { (controls) }\end{array}$ & $13.5(2.74-66.32)$ & 0.001 & $12.9(2.07-76.94)$ & 0.006 \\
\hline
\end{tabular}

Data are presented as OR $(95 \% \mathrm{Cl})$ for pulmonary hemorrhage for the binary exposures female gender, application of antenatal steroids, FFP

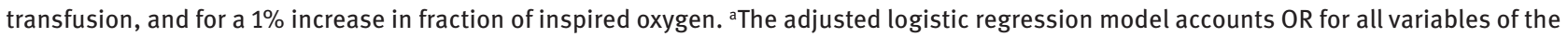
univariate model. $\mathrm{Cl}$, Confidence interval; FFP, fresh frozen plasma; OR, odds ratio. 
Our study underlines the beneficial effects of antenatal steroids beyond fetal lung maturation. As in previous studies [1, 2, 4], we found a protective effect of antenatal steroids for $\mathrm{PH}$ prevention. Antenatal steroids might not have been given due to the necessity of rapid delivery as a result of maternal illness or fetal distress. Although not reflected by higher CRIB scores, the reduced incidence of $\mathrm{PH}$ in response to steroids may result, on the cellular level, from enhanced microvascular maturation and premature focal capillary fusion [18]. The lower rate of fetal lung maturation is also reflected by the higher concentration of supplemental oxygen on the day of admission in infants with later $\mathrm{PH}$. However, at the time point of $\mathrm{PH}$, the ventilation mode did not differ between infants with $\mathrm{PH}$ and case-controls. Therefore, we assume that mechanical ventilation associated with micro trauma of the lung alone does not seem to be a major risk factor for PH. Severe IVH was detected in 10 out 11 infants after PH occurred and is thought to be a consequence of clinical deterioration during $\mathrm{PH}$. However, other bleedings were only detected in infants with $\mathrm{PH}$. Although small for gestational age (SGA) has been described as a risk factor for PH, and SGA infants are prone to coagulopathy [19], we did not find a correlation between SGA and the occurrence of PH.

The follow-up analysis of the trial on indomethacin prophylaxis in preterms (TIPP) indicated that prophylactic indomethacin reduced the rate of early serious $\mathrm{PH}$ [1]. Rates of infants with $\mathrm{PH}$ who had received prophylactic indomethacin were only $25 \%$ of those in controls, but this difference was not significant. In another recent casecontrol analysis, infants who received indomethacin were less likely to die, particularly in the $\mathrm{PH}$ group, in which moderate-to-large PDA was attributed to $\mathrm{PH}$ [2]. In order to avoid side effects, such as decreased cerebral blood flow, prophylactic indomethacin might be administered according to a risk score for major PH or IVH.

Although COX inhibitors, such as indomethacin and ibuprofen, are known to impair platelet function [20], their use for the prevention and therapy of PDA did not increase the occurrence of IVH in very low birth weight infants with platelet counts $>100 / \mathrm{nL}$ [21]. However, Brunner et al. showed that ibuprofen treatment significantly increased the risk for IVH in infants with platelet counts of 50-99/ $\mathrm{nL}$ [21]. In our study cohort, the rate of ibuprofen or indomethacin treatment did not differ in infants with $\mathrm{PH}$ and case-controls. Therefore, we assume that impaired platelet function due to COX inhibitor exposure does not play a significant, or plays only a minor, role in the pathophysiology of PH in non-thrombocytopenic infants.

Importantly, the implication of platelet and coagulation data on the individual risk for $\mathrm{PH}$ should be revisited for the following reasons: First, platelet counts and INR and fibrinogen concentration at birth did not differ between infants with $\mathrm{PH}$ and case-controls. The aPTT was significantly longer in infants with later $\mathrm{PH}$, and there was a trend towards a higher proportion of abnormal aPTT and fibrinogen concentration. This finding might explain the higher rate of FFP usage in infants with $\mathrm{PH}$. Second, platelet counts at onset of $\mathrm{PH}$ were lower than those at birth and also lower than the lowest values observed in controls during the first $96 \mathrm{~h}$ after birth. We assume that the decreased platelet count is a result of platelet consumption during acute hemorrhage. However, platelet counts $<50 / \mathrm{nL}$, widely thought to define severe thrombocytopenia, was seen not only in $10 \%$ of infants with $\mathrm{PH}$, but also in $7.5 \%$ of controls. This finding is in line with the outcome study on very low birth weight infants with platelet counts $<60 / \mathrm{nL}$ (PlaNet1), in which $9 \%$ of children developed major hemorrhage [5].

Finally, infants with $\mathrm{PH}$ received more transfusions of FFP than controls. FFP transfusion was an independent risk factor associated with $\mathrm{PH}$. It is tempting to speculate that the receipt of FFP is merely a marker of critical illness, but this cannot be concluded from other parameters such as requirement of mechanical ventilation, CRIB score, or surfactant treatment, as well as diagnosis of early-onset infection (Table 1). Thus, treatment with blood products deserves more attention. In 1996, a randomized controlled multicenter trial including 776 infants born before 32 weeks of gestation compared early FFP transfusion vs. maintenance glucose infusion (controls) with the primary outcome measure survival without disability. This trial provided no evidence that FFP transfusions affect the risks of death, IVH, or other major disability [22, 23]. Notably, the transfused plasma derived from adult donors may negatively interfere with neonatal platelet function, and hyporeactivity of neonatal platelets may be required for balancing primary hemostasis. Significant effects on platelet aggregation have been detected after 'developmental mismatch in vitro transfusions': the addition of adult platelets to cord blood plasma of thrombocytopenic neonates resulted in shorter clotting times, which in turn increased the bleeding risk as a consequence of prothrombotic effects. In the opposite approach, the addition of adult donor plasma to platelets of preterm infants led to prolonged bleeding time parameters. In line with this, no evidence is given that in preterm neonates, prophylactic plasma transfusion based on coagulation screens improved clinical outcomes in terms of mortality and morbidity [6, 23-25]. However, routine screening of coagulation in neonates leads to increased use of plasma for transfusion in neonatal intensive care [3]. Indeed, our 
analysis of blood coagulation screens did not indicate significant differences between ELBW infants with regard to fibrinogen and INR in infants with $\mathrm{PH}$ and age-matched controls. As indicated by prolonged aPTT in infants with later $\mathrm{PH}$ and occurrence of other bleeding symptoms, some patients might suffer from temporarily deranged coagulation, which contributes to the occurrence of $\mathrm{PH}$.

Several, somewhat obscure, findings might be of particular interest for further research on bleeding disorders of very premature infants. Platelet counts dropped significantly more often after birth in patients with $\mathrm{PH}$ than in controls. The exact time point of the decrease in platelet count can only be estimated between birth and onset of $\mathrm{PH}$. Platelet count might be decreased at onset of $\mathrm{PH}$ due to platelet consumption during clotting in the bleeding sites in the lung parenchyma. On the other hand, the platelets might be involved in the pathogenesis of $\mathrm{PH}$. Besides their primary role in coagulation, activated platelets mediate pulmonary neutrophil tethering and activation [26]. In the developmental-stage-dependent balanced coagulation system, changes in platelet counts and function (eventually related to their granula content, e.g. platelet-released Dickkopf-1 [Dkk1] or chemokines such as CCL5/RANTES $[27,28])$ could contribute to damage of the pulmonary endothelial barrier with subsequent bleeding. Such mechanisms for the response to local pulmonary inflammatory processes and other mechanisms for the augmented tension in the capillary wall (e.g. PDA, intrauterine growth restriction) deserve further experimental research in animal models for lung diseases in prematurity.

In summary, antenatal steroids are associated with a reduced risk for $\mathrm{PH}$. We cannot conclude whether the abnormal coagulation or the transfusion of FFP is causative for PH. However, as transfusion of adult plasma was associated with later $\mathrm{PH}$, clinical trials on laboratory based values as transfusion criteria are justified. In vivo experiments remain needed to understand suggested adverse effects on primary hemostasis by transfusing adult plasma to ELBW infants.

Acknowledgement: We thank Boris Metze, BSc, for his help in obtaining the clinical data and performing statistical analysis, Petra Blank, Jessica Blank, and Regina Nagel for administrative support, and Karine Landgren Hugentobler for language editing.

Contributors: $\mathrm{CD}, \mathrm{LG}, \mathrm{CB}$, and MC conceived the study. Clinical data was analyzed by JU; statistics were done by JU and MC. JU, MC, and CD drafted the article, and all authors agreed to the final manuscript.

Funding: None.

\section{Author's Statement}

Conflict of interest: The authors declare that there are no conflicts of interest regarding the publication of this article.

Material and methods: Informed consent: Informed consent has been obtained from all individuals included in this study.

Ethical approval: The study was approved by the local Institutional Review Board (Ethikkommission der Charité - Universitätsmedizin Berlin) that waived the need to obtain informed parental consent.

\section{References}

[1] Alfaleh K, Smyth JA, Roberts RS, Solimano A, Asztalos EV, Schmidt B, et al. Prevention and 18-month outcomes of serious pulmonary hemorrhage in extremely low birth weight infants: results from the trial of indomethacin prophylaxis in preterms. Pediatrics. 2008;121:e233-8.

[2] Scholl JE, Yanowitz TD. Pulmonary hemorrhage in very low birth weight infants: a case-control analysis. J Pediatr. 2015;166:1083-4.

[3] Catford K, Muthukumar P, Reddy C, Al Atrash H, Clarke P, Venkatesh V, et al. Routine neonatal coagulation testing increases use of fresh-frozen plasma. Transfusion. 2014;54:1444-5.

[4] Berger TM, Allred EN, Van Marter LJ. Antecedents of clinically significant pulmonary hemorrhage among newborn infants. J Perinatol. 2000;20:295-300.

[5] Stanworth SJ, Clarke P, Watts T, Ballard S, Choo L, Morris T, et al. Prospective, observational study of outcomes in neonates with severe thrombocytopenia. Pediatrics. 2009;124:e826-34.

[6] Christensen RD, Baer VL, Lambert DK, Henry E, Ilstrup SJ, Bennett ST. Reference intervals for common coagulation tests of preterm infants (CME). Transfusion. 2014;54:627-32:quiz 6.

[7] Duppre P, Sauer H, Giannopoulou EZ, Gortner L, Nunold H, Wagenpfeil S, et al. Cellular and humoral coagulation profiles and occurrence of IVH in VLBW and ELWB infants. Early Hum Dev. 2015;91:695-700.

[8] Batfalsky A, Lohr A, Heussen N, Neunhoeffer F, Orlikowsky TW. Diagnostic value of an interleukin- 6 bedside test in term and preterm neonates at the time of clinical suspicion of early- and late-onset bacterial infection. Neonatology. 2012;102:37-44.

[9] Papile LA, Burstein J, Burstein R, Koffler H. Incidence and evolution of subependymal and intraventricular hemorrhage: a study of infants with birth weights less than 1,500 gm. J Pediatr. 1978;92:529-34.

[10] Schmidt B, Roberts RS, Fanaroff A, Davis P, Kirpalani HM, Nwaesei $C$, et al. Indomethacin prophylaxis, patent ductus arteriosus, and the risk of bronchopulmonary dysplasia: further analyses from the Trial of Indomethacin Prophylaxis in Preterms (TIPP). J Pediatr. 2006;148:730-4.

[11] Fowlie PW. Intravenous indomethacin for preventing mortality and morbidity in very low birth weight infants. Cochrane Database Syst Rev. 2000:CD000174. 
[12] Heuchan AM, Evans N, Henderson Smart DJ, Simpson JM. Perinatal risk factors for major intraventricular haemorrhage in the Australian and New Zealand Neonatal Network, 1995-97. Arch Dis Child Fetal Neonatal Ed. 2002;86:F86-90.

[13] Ohlsson A, Roberts RS, Schmidt B, Davis P, Moddeman D, Saigal $S$, et al. Male/female differences in indomethacin effects in preterm infants. J Pediatr. 2005;147:860-2.

[14] Leduc L, Takser L, Rinfret D. Persistance of adverse obstetric and neonatal outcomes in monochorionic twins after exclusion of disorders unique to monochorionic placentation. Am J Obstet Gynecol. 2005;193:1670-5.

[15] Kassal R, Anwar M, Kashlan F, Smulian J, Hiatt M, Hegyi T. Umbilical vein interleukin-6 levels in very low birth weight infants developing intraventricular hemorrhage. Brain Dev. 2005;27:483-7.

[16] Roberts IA, Murray NA. Thrombocytopenia in the newborn. Curr Opin Pediatr. 2003;15:17-23.

[17] Abou Zahr R, Ashfaq A, Marron-Corwin M. Neonatal pulmonary hemorrhage. NeoReviews. 2012;13:e302-6.

[18] Roth-Kleiner M, Berger TM, Tarek MR, Burri PH, Schittny JC. Neonatal dexamethasone induces premature microvascular maturation of the alveolar capillary network. Dev Dyn. 2005;233:1261-71.

[19] Hannam S, Lees C, Edwards RJ, Greenough A. Neonatal coagulopathy in preterm, small-for-gestational-age infants. Biol Neonate. 2003;83:177-81.

[20] De la Cruz JP, Reyes JJ, Ruiz-Moreno MI, Lopez-Villodres JA, Jebrouni N, Gonzalez-Correa JA. Differences in the in vitro antiplatelet effect of dexibuprofen, ibuprofen, and flurbiprofen in human blood. Anesth Analg. 2010;111:1341-6.

[21] Brunner B, Hoeck M, Schermer E, Streif W, Kiechl-Kohlendorfer U. Patent ductus arteriosus, low platelets, cyclooxygenase inhibitors, and intraventricular hemorrhage in very low birth weight preterm infants. J Pediatr. 2013;163:23-8.

[22] Randomised trial of prophylactic early fresh-frozen plasma or gelatin or glucose in preterm babies: outcome at 2 years. Northern Neonatal Nursing Initiative Trial Group. Lancet. 1996;348:229-32.

[23] A randomized trial comparing the effect of prophylactic intravenous fresh frozen plasma, gelatin or glucose on early mortality and morbidity in preterm babies. The Northern Neonatal Nursing Initiative [NNNI] Trial Group. Eur J Pediatr. 1996;155:580-8.

[24] Kelly AM, Williamson LM. Neonatal transfusion. Early Hum Dev. 2013;89:855-60.

[25] Pal S, Curley A, Stanworth SJ. Interpretation of clotting tests in the neonate. Arch Dis Child Fetal Neonatal Ed. 2015;100:F270-4.

[26] Semple JW, Italiano JE, Jr., Freedman J. Platelets and the immune continuum. Nat Rev Immunol. 2011;11:264-74.

[27] Guo Y, Mishra A, Howland E, Zhao C, Shukla D, Weng T, et al. Platelet-derived Wnt antagonist Dickkopf-1 is implicated in ICAM-1/VCAM-1-mediated neutrophilic acute lung inflammation. Blood. 2015;126:2220-9.

[28] Hwaiz R, Rahman M, Syk I, Zhang E, Thorlacius H. Rac1-dependent secretion of platelet-derived CCL5 regulates neutrophil recruitment via activation of alveolar macrophages in septic lung injury. J Leukoc Biol. 2015;97:975-84. 